\title{
On the Design of Multiuser Codebooks for Uplink SCMA Systems
}

\author{
Jinchen Bao, Zheng Ma, Member, IEEE, Zhiguo Ding, Senior Member, IEEE, \\ George K. Karagiannidis, Fellow, IEEE, and Zhongliang Zhu
}

\begin{abstract}
Sparse code multiple access (SCMA) is a promising uplink multiple access technique that can achieve superior spectral efficiency, provided that multidimensional codebooks are carefully designed. In this letter, we investigate the multiuser codebook design for SCMA systems over Rayleigh fading channels. The criterion of the proposed design is derived from the cutoff rate analysis of the equivalent multiple-input multipleoutput system. Furthermore, new codebooks with signal-space diversity are suggested, while simulations show that this criterion is efficient in developing codebooks with substantial performance improvement, compared with the existing ones.
\end{abstract}

Index Terms-Sparse code multiple access (SCMA), multidimensional constellations, cutoff rate, signal-space diversity.

\section{INTRODUCTION}

$\mathbf{S}$ PARSE code multiple access (SCMA) is a code/frequence domain non-orthogonal multiple access technique, proposed for the fifth generation $(5 \mathrm{G})$ wireless networks [1]-[6]. It is able to provide good spectrum efficiency due to the use of multidimensional complex codebooks/constellations. However, the joint design of multiple multidimensional codebooks is generally challenging. Scanning the open literature, the design of SCMA codebooks are limited to a few works, while - to the best of the authors' knowledge - the optimal design criterion for multiuser codebooks is unknown. More specifically, Nikopour and Baligh [1] and Taherzadeh et al. [4] proposed a unified framework, where a multi-stage suboptimal approach is used, for the joint design of multiuser codebooks. Following this direction, several methods were proposed to increase the coding gain of SCMA codebooks, or to lower the complexity of multiuser detection. These approaches include constellation with low projections [4], [5], spherical codebooks [7], dimensional rotation and interleave [8] and starQAM based multidimensional signaling [9]. However, they only provide some rough insights on the multiuser codebooks, and an efficient design criterion was not introduced.

Manuscript received July 12, 2016; revised July 28, 2016; accepted July 28, 2016. This work is supported by Huawei HIRP project (no.YB201504), National Nature Science Foundation of China under Grant 61571373, Fundamental Research Funds for the Central Universities (no. 2682016ZDPY06). The associate editor coordinating the review of this paper and approving it for publication was A. Lioumpas.

J. Bao, Z. Ma, and Z. Zhu are with the Provincial Key Laboratory of Information Coding and Transmission, Southwest Jiaotong University, Chengdu 611756, China (e-mail: jinchen_bao@my.swjtu.edu.cn; zma@home.swjtu.edu.cn; jczuoyi@163.com).

Z. Ding is with the School of Computing and Communications, Lancaster University, Lancaster LA1 4YW, U.K. (e-mail: z.ding@lancaster.ac.uk).

G. K. Karagiannidis is with the Provincial Key Laboratory of Information Coding and Transmission, Southwest Jiaotong University, Chengdu 611756, China, and also with the Electrical and Computer Engineering Department, Aristotle University of Thessaloniki, Thessaloniki 54124, Greece (e-mail: geokarag@auth.gr).

Digital Object Identifier 10.1109/LCOMM.2016.2596759

In this letter, a novel and efficient performance criterion is proposed, for the joint design of multiuser codebooks in uplink SCMA systems. The basic idea is to optimize the mutual information and cutoff rate of the equivalent multiple input multiple output (MIMO) system. Based on this criterion, we propose a rotated multidimensional constellation with signalspace diversity in order to choose the multiuser constellations. In addition, we investigate the applications of the proposed codebooks in a coded system with iterative multiuser detection [6], through an extrinsic information transfer (EXIT) chart analysis. Simulation results verify the efficiency of the proposed codebook design and the significant achieved performance gain, compared to other existing methods.

\section{SySTEM MODEL}

We consider $J$ users/layers employing $K$-dimensional constellations transmitting over a multiple access channel, where each user employs only one layer. The message, which carries a data stream in bits for each user, is encoded and mapped into a $K$-dimensional symbol, $\mathbf{x}_{j}$. Then, each symbol is transmitted over $K$ resources, such as time slots or orthogonal frequency division multiplexing (OFDM) tones, without inter-carrier interference. At the receiver, the $K$-dimensional channel output vector, $\mathbf{y}=[y[1], \cdots, y[K]]^{t}$, is expressed as

$$
\mathbf{y}=\sum_{j=1}^{J} \operatorname{diag}\left(\mathbf{h}_{j}\right) \mathbf{x}_{j}+\mathbf{n}=\mathbf{H X}+\mathbf{n},
$$

where the $\operatorname{diag}(\mathbf{h})$, which denotes the diagonal matrix with its $k$-th diagonal element, corresponds to the $k$-th entry of vector $\mathbf{h}$, and $[\cdot]^{t}$ denotes transpose. The $K \times 1$ data symbol $\left(\mathbf{x}_{j}\right)$, the $K \times 1$ additive white Gaussian noise (AWGN) vector with independent and identically distributed (i.i.d) components of zero mean and variance $\frac{N_{0}}{2}$ per dimension, and the $K \times 1$ channel gain vector, $\mathbf{h}_{j}$, between the $j$-th user and the receiver are given by

$$
\mathbf{x}_{j}=\left[\begin{array}{c}
x_{j}[1] \\
\vdots \\
x_{j}[K]
\end{array}\right], \quad \mathbf{n}=\left[\begin{array}{c}
n[1] \\
\vdots \\
n[K]
\end{array}\right], \quad \mathbf{h}_{j}=\left[\begin{array}{c}
h_{j}[1] \\
\vdots \\
h_{j}[K]
\end{array}\right] .
$$

The equivalent channel matrix $\mathbf{H}$ with size $K \times K J$ is given by

$$
\mathbf{H}=\left[\operatorname{diag}\left(\mathbf{h}_{1}\right), \operatorname{diag}\left(\mathbf{h}_{2}\right), \cdots, \operatorname{diag}\left(\mathbf{h}_{J}\right)\right]_{K \times K J},
$$

where $\mathbf{X}=\left[\mathbf{x}_{1}^{t}, \mathbf{x}_{2}^{t}, \cdots, \mathbf{x}_{J}^{t}\right]^{t}$ is the combined transmitted signal vector for $J$ users. Therefore, the multiple access channel model can be viewed as a MIMO communication system with $K J$ inputs and $K$ outputs. The discrete input to the equivalent MIMO channel is a combined constellation, which can be represented as, $\tilde{X}=x_{1} \times \cdots \times x_{J}$, where $x_{j}$ 
is the constellation of the $j$-th user, and it is assumed that its norm, $\left|x_{j}\right|=M$, for all $j$.

The sparseness of codewords can be completely characterized by a factor graph matrix [1]. Denote $\phi_{k}=$ $\left\{j: 1 \leq j \leq J, x_{j}[k] \neq 0\right\}$, the index of users that contribute to the $k$-th resource, then the cardinality of $\phi_{k}$ is equal to the row weight of the $k$-th row in this matrix.

\section{Mutual Information Analysis}

Assuming that perfect channel knowledge is available at the receiver. The mutual information $I(\mathbf{X} ; \mathbf{y})$ between the discrete input $\mathbf{X}$ and the continuous output $\mathbf{y}$, conditioned on the fixed channel matrix $\mathbf{H}$, is given by

$I(\mathbf{X} ; \mathbf{y} \mid \mathbf{H})$

$$
\begin{aligned}
= & \log C-\frac{1}{C} \sum_{\mathbf{X}_{a}} \frac{1}{\left(\pi N_{0}\right)^{K}}\left\{\int_{\mathbf{y}} \exp \left(-\frac{1}{N_{0}}\left\|\mathbf{y}-\mathbf{H} \mathbf{X}_{a}\right\|^{2}\right)\right. \\
& \left.\times \log \left[\sum_{\mathbf{x}_{b}, b \neq a} \exp \left(\frac{\left\|\mathbf{y}-\mathbf{H} \mathbf{X}_{a}\right\|^{2}-\left\|\mathbf{y}-\mathbf{H} \mathbf{X}_{b}\right\|^{2}}{N_{0}}\right)\right] d \mathbf{y}\right\},
\end{aligned}
$$

where $C=M^{J}$, is the number of all possible inputs to the equivalent channel, and $\mathbf{X}_{a}, \mathbf{X}_{b} \in \tilde{\chi}$ are two distinct symbols from the combined constellation. The average mutual information can be obtained by averaging over the distribution of $\mathbf{H}$.

Since it is quite difficult-if not impossible-to deal with an expression for the mutual information, we consider the cutoff rate [10], instead. The conditional cutoff rate for a given channel gain $\mathbf{H}$ is defined as

$R(\mathbf{X} ; \mathbf{y} \mid \mathbf{H})$

$$
=\log C-\log \left[1+\frac{1}{C} \sum_{\mathbf{X}_{a}} \sum_{\mathbf{X}_{b}, b \neq a} \exp \left(-\frac{\left\|\mathbf{H}\left(\mathbf{X}_{a}-\mathbf{X}_{b}\right)\right\|^{2}}{4 N_{0}}\right)\right] .
$$

Let us define

$$
\begin{aligned}
\Psi\left(\mathbf{X}_{a}, \mathbf{X}_{b}, \mathbf{H}\right) & =\exp \left(-\frac{\left\|\mathbf{H}\left(\mathbf{X}_{a}-\mathbf{X}_{b}\right)\right\|^{2}}{4 N_{0}}\right) \\
& =\exp \left(-\frac{\sum_{k=1}^{K}\left\|\mathbf{h}[k]^{\dagger}\left(\mathbf{x}_{a}[k]-\mathbf{x}_{b}[k]\right)\right\|^{2}}{4 N_{0}}\right),
\end{aligned}
$$

where $\mathbf{x}_{a}[k]=\left[x_{1, a}[k], \cdots, x_{J, a}[k]\right]^{t}$, is the vector of the $k$-th component for $J$ users, and $\mathbf{h}[k]^{\dagger}=\left[h_{1}[k], \cdots, h_{J}[k]\right]$, are the corresponding channel gains, and $[\cdot]^{\dagger}$ denotes conjugate transpose.

Using the matrix decomposition, it holds that

$$
\left(\mathbf{x}_{a}[k]-\mathbf{x}_{b}[k]\right)\left(\mathbf{x}_{a}[k]-\mathbf{x}_{b}[k]\right)^{\dagger}=\mathbf{U}_{k} \Lambda_{k} \mathbf{U}_{k}^{\dagger},
$$

where $\mathbf{U}_{k}$ is unitary and $\Lambda_{k}$ is a diagonal matrix, i.e. $\Lambda_{k}=$ $\operatorname{diag}\left(\lambda_{k, 1}^{2}, \cdots, \lambda_{k, J}^{2}\right)$, with $\lambda_{k, j}^{2}$ being the ordered eigenvalues of the matrix $\left(\mathbf{x}_{a}[k]-\mathbf{x}_{b}[k]\right)\left(\mathbf{x}_{a}[k]-\mathbf{x}_{b}[k]\right)^{\dagger}$. Hence,

$$
\begin{aligned}
\left\|\mathbf{h}[k]^{\dagger}\left(\mathbf{x}_{a}[k]-\mathbf{x}_{b}[k]\right)\right\|^{2} & =\mathbf{h}[k]^{\dagger} \mathbf{U}_{k} \Lambda_{k} \mathbf{U}_{k}^{\dagger} \mathbf{h}[k] \\
& =\hat{\mathbf{h}}[k]^{\dagger} \Lambda_{k} \hat{\mathbf{h}}[k]=\lambda_{k}^{2}\left|\hat{h}_{1}[k]\right|^{2},
\end{aligned}
$$

where we define, $\hat{\mathbf{h}}[k]^{\dagger}=\mathbf{h}[k]^{\dagger} \mathbf{U}_{k}$. Thus, $\hat{\mathbf{h}}[k]$ has the same distribution as $\mathbf{h}[k]$, and $\lambda_{k}^{2}=\left\|\mathbf{x}_{a}[k]-\mathbf{x}_{b}[k]\right\|^{2}$, is the unique nonzero singular value in $\Lambda_{k}$ (the index 1 is dropped here for $\left.\lambda_{k, 1}^{2}\right)$. Due to the sparseness of the codebooks,

$$
\lambda_{k}^{2}=\sum_{j \in \phi_{k}}\left|x_{j, a}[k]-x_{j, b}[k]\right|^{2} .
$$

Therefore, it holds that

$$
\Psi\left(\mathbf{X}_{a}, \mathbf{X}_{b}, \mathbf{H}\right)=\prod_{k=1}^{K} \exp \left(-\frac{\lambda_{k}^{2}\left|\hat{h}_{1}[k]\right|^{2}}{4 N_{0}}\right) .
$$

Averaging over the distribution of $\mathbf{H}$ and assuming independent Rayleigh fadings, results in

$$
\mathbb{E}_{\mathbf{H}}\left[\Psi\left(\mathbf{X}_{a}, \mathbf{X}_{b}, \mathbf{H}\right)\right]=\prod_{k=1}^{K}\left(1+\frac{\lambda_{k}^{2}}{4 N_{0}}\right)^{-1},
$$

where $\mathbb{E}[\cdot]$ denotes the mean. Thus, the mean cutoff rate is given by

$$
\begin{aligned}
R(\mathbf{X} ; \mathbf{y}) & =\mathbb{E}_{\mathbf{H}}[R(\mathbf{X} ; \mathbf{y} \mid \mathbf{H})] \\
& \geq \log C-\log \left(1+\frac{1}{C} \sum_{\mathbf{X}_{a}} \sum_{\mathbf{X}_{b}, b \neq a} \mathbb{E}_{\mathbf{H}}\left[\Psi\left(\mathbf{X}_{a}, \mathbf{X}_{b}, \mathbf{H}\right)\right]\right) \\
& =\log C-\log \left[1+\frac{1}{C} \sum_{\mathbf{X}_{a}} \sum_{\mathbf{X}_{b}, b \neq a} \prod_{k=1}^{K}\left(1+\frac{\lambda_{k}^{2}}{4 N_{0}}\right)^{-1}\right] .
\end{aligned}
$$

The first step above follows from the Jensen inequality.

It should be noted that the term in the square bracket of (12) is the union bound for the joint symbol error probability (SEP) for multiple users in i.i.d Rayleigh fading, which means that optimizing the mean cutoff rate is equivalent to the optimization of the average SEP. Therefore, we can formulate the cutoff rate criterion for the multiuser codebooks design, by making the union bound on the average SEP as small as possible.

\section{Design Multiuser Codebooks}

According to the analysis in the previous section, the goal is to design the constellation $\tilde{X}$, which maximizes the cutoff rate of the equivalent MIMO system. From (12), maximizing $R(\mathbf{X} ; \mathbf{y})$ is equivalent to choose the combined constellation such that

$$
\begin{aligned}
& \tilde{X}=\arg \min _{\tilde{\tilde{x}}} \sum_{\mathbf{X}_{a}} \sum_{\mathbf{X}_{b}, b \neq a} \prod_{k=1}^{K}\left(1+\frac{\lambda_{k}^{2}}{4 N_{0}}\right)^{-1} \\
& \text { s.t. } \mathbb{E}\left[\|\mathbf{X}\|^{2}\right]=E_{S},
\end{aligned}
$$

where $E_{s}$ is the average power. It is expected that the criterion is optimal in the sense of designing codebooks with large mutual information, since it involves all pairs of possible symbols for multiple users.

Recall that the problem of designing multidimensional constellations for fading channels has been solved by using signalspace diversity, which rotates the QAM constellations with a unitary matrix, constructed either from the algebraic number theory or by computer search [11], [12]. Therefore, in this 
work, we use the rotated constellation to build the multiuser codebooks. In particular, we obtain the rotation matrices through computer search over compact parameterizations of unitary matrices. Note, that the null dimensions of codebooks are discarded before the rotations. The $N \times N$ unitary matrix can be written as [12]

$$
\mathbf{R}=\prod_{m=1}^{N-1} \prod_{n=m+1}^{N} \mathbf{T}_{m n},
$$

where $\mathbf{T}_{m n}$ is a complex Givens matrix [13], which changes the identity matrix by replacing its $(m, m)$ th, $(n, n)$ th, $(m, n)$ th and $(n, m)$ th elements with $\cos \theta_{m n}, \cos \theta_{m n}, e^{-i \eta_{m n}} \sin \theta_{m n}$ and $-e^{i \eta_{m n}} \sin \theta_{m n}$, respectively. The angles satisfy $\theta_{m n} \in$ $[-\pi, \pi]$ and $\eta_{m n} \in[-\pi / 2, \pi / 2]$. So, the search for $\mathbf{R}$ is reduced to the search for a sequence of phase of

$$
\begin{aligned}
& \boldsymbol{\theta}=\left\{\theta_{1,2}, \cdots, \theta_{1, N}, \theta_{2,3}, \cdots, \theta_{2, N}, \cdots, \theta_{N-1, N}\right\}, \\
& \boldsymbol{\eta}=\left\{\eta_{1,2}, \cdots, \eta_{1, N}, \eta_{2,3}, \cdots, \eta_{2, N}, \cdots, \eta_{N-1, N}\right\} .
\end{aligned}
$$

It seems that optimizing the constellations using the above criterion is intractable even for a moderate number of users and codebook size, since we need to search $N(N-1) J$ angles, and the summation in the right hand side of (13) will add up $C(C-1)$ terms. However, searching results for twodimensional constellations with a small number of users show that the rotation matrices are the same for all codebooks, and are independent of the number of users. Therefore, we simplify the optimization process by searching over a single rotation matrix, even though this is suboptimal. Furthermore, we can use the approach developed in [14], where all the entries of the rotation matrix are equal in magnitude. Therefore, by expanding the product in (14), we get $\boldsymbol{\theta}=\left\{\frac{\pi}{4}\right\}$ and $\theta=\left\{\frac{\pi}{4}, 0.6155, \frac{\pi}{4}\right\}$ for $N=2$ and $N=3$, respectively. Exhaustive search is computationally feasible, provided, that each user occupies a moderate number of resources such that $N \leq 3$.

\section{Simulations AND Discussions}

We investigate the block error rate (BLER) performance for an uncoded SCMA system with receive diversity, with two antennas. The message passing algorithm (MPA) detector is used with 6 iterations all the time. The SCMA system follows a factor graph matrix as in (16),

$$
\mathbf{F}=\left[\begin{array}{llllll}
1 & 0 & 1 & 0 & 1 & 0 \\
0 & 1 & 1 & 0 & 0 & 1 \\
1 & 0 & 0 & 1 & 0 & 1 \\
0 & 1 & 0 & 1 & 1 & 0
\end{array}\right],
$$

which occupies four resources and affords up to 6 users and a maximum overloading factor $150 \%(J / K)$. The simulations are performed over i.i.d Rayleigh fading channels for 4-ary and 16-ary codebooks, where the codebook from [4] and spherical codes [7] are used as the benchmark. We also provide the results of the star-QAM based codebooks [9] after optimization using our approach, for which we extend $\alpha$ to complex numbers and get $\beta=1, \alpha=-i$ and $\alpha=0.8-0.8 i$ for 4-ary and 16-ary codebooks, respectively. ${ }^{1}$

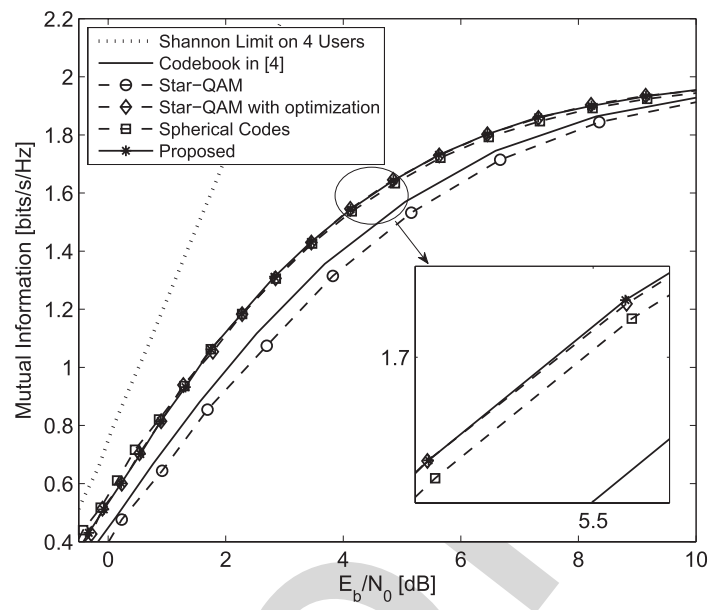

Fig. 1. Mutual information of 4-ary SCMA codebooks for 4 users.

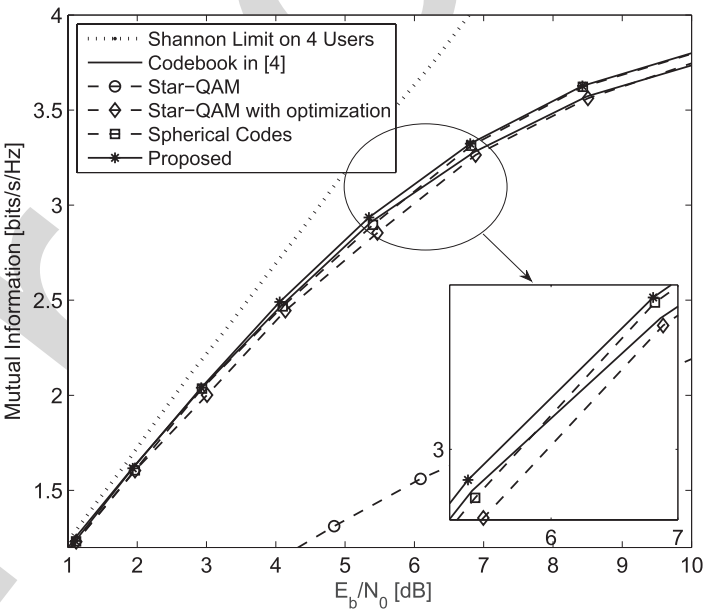

Fig. 2. Mutual information of 16-ary SCMA codebooks for 4 users.

Figs. 1 and 2 plot the codebook constraint capacities of ${ }_{211}$ 4-ary and 16-ary codebooks for 4 users with one receiving ${ }_{212}$ antenna, together with the theoretical limit rates of i.i.d Gaussian inputs. As it is evident, the proposed scheme outperforms all the other codebooks in the high rate region for both 4-ary and 16-ary codebooks, while the mutual information gain is more clear for 4-ary case. While the rate of the starQAM scheme is quite small, a significant gain is achieved after optimization with our criterion, and it becomes as good as the proposed one for 4-ary codebook.

Figs. 3 and 4 compare the BLER performance of the proposed scheme with existing ones for 4-ary and 16-ary codeboks, respectively. As it is observed, the proposed codebook has a gain about $0.8 \mathrm{~dB}$ and $0.6 \mathrm{~dB}$ over that in [4] for 4-ary and 16-ary cases, and a gain about $0.5 \mathrm{~dB}$ and $0.3 \mathrm{~dB}$ over the spherical codes, respectively. Without optimization, the starQAM scheme yields the worst error performance. However, it performs much better after the optimization, which coincides with the result of mutual information in Figs. 1 and 2.

The iterative multiuser receiver [6] was introduced for SCMA, because of its low-complexity feature and its good performance. To examine whether the proposed codebook works

\footnotetext{
${ }^{1}$ The star-QAM based codebook targets on downlink channels, while its performance deteriorates in the uplink and for large constellation size.
} 


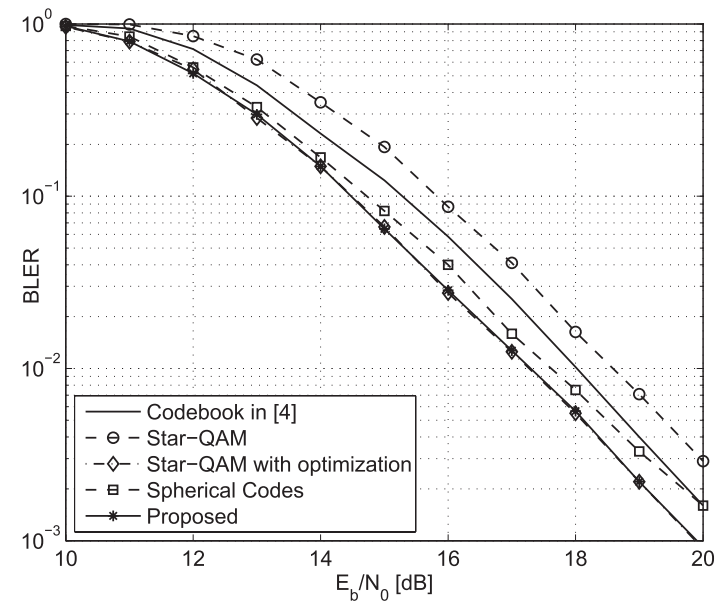

Fig. 3. BLER of 4-ary codebooks for uplink SCMA systems with 6 users over Rayleigh fading channels.

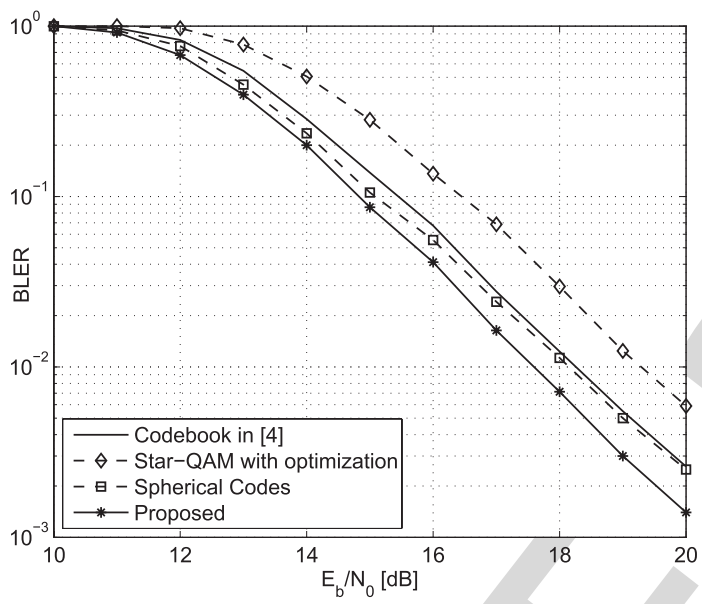

Fig. 4. BLER of 16-ary codebooks for uplink SCMA systems with 6 users over Rayleigh fading channels.

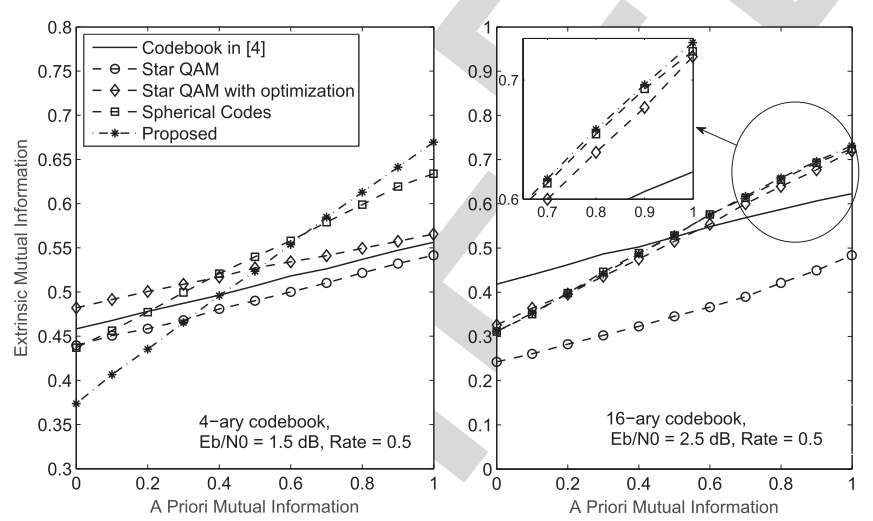

Fig. 5. Exit chart curve of MPA detector for 6 users over Rayleigh fading channels. largest extrinsic information when ideal a priori information is available. If an appropriate outer code is designed to match the EXIT curves, more reliable detection is possible and a larger iterative gain can be achieved for the proposed codebook, compared to other ones. Note that the EXIT curves do not necessarily to match the result of uncoded performance, since in addition to the constellations, the labeling also plays an important role in an iterative decoding scenarios.

\section{CONCLUSions}

A performance criterion for the joint design of multiuser codebooks for uplink SCMA systems over Rayleigh fading channels was proposed, based on the cutoff rate of the equivalent MIMO system. The provided analysis showed that the used criterion significantly affects the average SEP. Based on this criterion, a codebook design with signal-space diversity, was proposed. Simulations showed that the proposed scheme provides substantial performance improvement compared to existing codebooks.

\section{REFERENCES}

[1] H. Nikopour and H. Baligh, "Sparse code multiple access," in Proc IEEE 24th Int. Symp. Pers., Indoor Mobile Radio Commun. (PIMRC), London, U.K., Sep. 2013, pp. 332-336.

[2] H. Nikopour et al., "SCMA for downlink multiple access of 5G wireless networks," in Proc. IEEE Global Commun. Conf. (GLOBECOM), Austin, TX, USA, Dec. 2014, pp. 3940-3945.

[3] S. Zhang, X. Xu, L. Lu, Y. Wu, G. He, and Y. Chen, "Sparse code multiple access: An energy efficient uplink approach for $5 \mathrm{G}$ wireless systems," in Proc. IEEE Global Commun. Conf. (GLOBECOM), Austin, TX, USA, Dec. 2014, pp. 4782-4787.

[4] M. Taherzadeh, H. Nikopour, A. Bayesteh, and H. Baligh, "SCMA codebook design," in Proc. IEEE 80th Veh. Technol. Conf. (VTC Fall), Vancouver, BC, Canada, Sep. 2014, pp. 1-5.

[5] A. Bayesteh, H. Nikopour, M. Taherzadeh, H. Baligh, and J. Ma, "Low complexity techniques for SCMA detection," in Proc. IEEE Globecom Workshops, San Diego, CA, USA, Dec. 2015, pp. 1-6.

[6] Y. Wu, S. Zhang, and Y. Chen, "Iterative multiuser receiver in sparse code multiple access systems," in Proc. IEEE Int. Commun. Conf. (ICC), London, U.K., Jun. 2015, pp. 2918-2923.

[7] J. Bao, Z. Ma, M. A. Mahamadu, Z. Zhu, and D. Chen, "Spherical codes for SCMA codebook," in Proc. IEEE 83rd Veh. Technol. Conf. (VTC Spring), May 2016, pp. 1-5.

[8] D. Cai, P. Fan, X. Lei, Y. Liu, and D. Chen, "Multi-dimensional SCMA codebook design based on constellation rotation and interleaving," in Proc. IEEE 83rd Veh. Technol. Conf. (VTC Spring), May 2016, pp. 1-5.

[9] L. Yu, X. Lei, P. Fan, and D. Chen, "An optimized design of SCMA codebook based on star-QAM signaling constellations," in Proc. Int Conf. Wireless Commun. Signal Process. (WCSP), Nanjing, China, Oct. 2015, pp. 1-5.

[10] A. O. Hero and T. L. Marzetta, "Cutoff rate and signal design for the quasi-static Rayleigh-fading space-time channel," IEEE Trans. Inf. Theory, vol. 47, no. 6, pp. 2400-2416, Sep. 2001.

[11] J. Boutros and E. Viterbo, "Signal space diversity: A power- and bandwidth-efficient diversity technique for the Rayleigh fading channel," IEEE Trans. Inf. Theory, vol. 44, no. 4, pp. 1453-1467, Jul. 1998.

[12] Y. Xin, Z. Wang, and G. B. Giannakis, "Space-time diversity system based on linear constellation precoding," IEEE Trans. Wireless Commun., vol. 2, no. 2, pp. 294-309, Mar. 2003.

[13] G. H. Golub and C. F. Van Loan, Matrix Computations, 3rd ed. Baltimore, MD, USA: The Johns Hopkins Univ. Press, 1996.

[14] S. P. Herath, N. H. Tran, and T. Le-Ngoc, "Rotated multi-D constellations in Rayleigh fading: Mutual information improvement and pragmatic approach for near-capacity performance in high-rate regions,", IEEE Trans. Commun., vol. 60, no. 12, pp. 3694-3704, Dec. 2012.

[15] S. ten Brink, "Convergence behavior of iteratively decoded parallel concatenated codes," IEEE Trans. Commun., vol. 49, no. 10, pp. 1727-1737, Oct. 2001 . 